\title{
Restoring Balance and Symmetry to the Aging Nose
}

\author{
Samit N. Unadkat, FRCS (ORL-HNS) ${ }^{1 \odot}$ Hesham A. Saleh, FRCSI, FRCS (ORL-HNS), IBCFPRS 2,3 \\ ${ }^{1}$ Department of Rhinology \& Facial Plastic Surgery, Royal National \\ ENT \& University College Hospital, London, United Kingdom \\ 2 Department of Rhinology \& Facial Plastic Surgery, Charing Cross \\ Hospital, London, United Kingdom

\begin{abstract}
Address for correspondence Hesham A. Saleh, FRCSI, FRCS (ORLHNS), IBCFPRS, Department of Otolaryngology, Charing Cross Hospital, Imperial College London, Fulham Palace Road, London W6 8RF, United Kingdom (e-mail: hesham.saleh@btinternet.com).
\end{abstract}

3 Department of Rhinology, Imperial College London, London, United Kingdom of Great Britain and Northern Ireland

Facial Plast Surg 2021;37:205-210.

\begin{abstract}
Keywords

- aging

- face

- nasal septum

- surgical procedures

- rhinoplasty

Accurate assessment of facial symmetry is a key component of successful rhinoplasty surgery but is often overlooked by both surgeon and patient. Up to three-quarters of patients with a significantly crooked nose have been found to have concurrent marked facial asymmetry. Whilst not a contraindication to rhinoplasty, provided that facial asymmetry is identified in advance and expectations realistic, the correction of nasal deformities can improve perceived facial asymmetry. In the aging face, aside from soft tissue and bony resorption that can amplify facial asymmetry, there are specific surgical challenges to the aging nose; the normal tip support mechanisms deteriorate with age giving rise to distinctive changes to the aging nose-typically tip ptosis and a hanging columella; bone quality becomes more brittle and skin overlying this area becomes thinner. There is also weakening of the external nasal valve thus causing functional impairment too. As a result, rhinoplasty techniques used in younger patients may not be suitable in the aging nose. In this article, the authors describe their experience and outline the evidence on the management of the aging patient with facial and nasal asymmetry.
\end{abstract}

It has long been accepted that the proportions and symmetry of facial structures are key elements in the estimation of facial attractiveness. ${ }^{1,2}$ As the central structure of the face, the nose plays a particularly key role, not only through the perception of a crooked nose but also through its role in mid-facial growth. ${ }^{3}$ Indeed evolutionary biologists have even suggested that facial symmetry is a hallmark of health and genetic quality. ${ }^{4}$ From early on human infants pick up on even subtle facial asymmetries. More significant facial asymmetries are known to be associated with specific neurological conditions such as Down's syndrome, Fragile X, and velo-cardio-facial syndromes. ${ }^{4}$

Rhinoplasty surgery remains in the top five most performed aesthetic surgical procedures worldwide ${ }^{5}$ and yet repeatedly, underlying facial asymmetries are often overlooked, by both surgeon and patient. Accurate assessment of any underlying facial asymmetry is crucial to preoperative rhinoplasty planning.

There have been many published studies in the literature that have positively demonstrated the correlation between nasal deviation and facial asymmetry. ${ }^{1,6}$ According to Yi and Jang, in patients without a history of nasal trauma, the nasal axis will typically drift to the smaller side of the face and be accompanied by chin tilt. ${ }^{1}$ Thus, for optimum outcomes in rhinoplasty surgery, it is not only the externally deviated nose that requires correction, one must also take into consideration the nasal septum. Add to that patient expectations and this makes rhinoplasty surgery the most challenging of facial aesthetic procedures.

Rhinoplasty in the aging population carries an additional challenge compared to the traditional rhinoplasty-seeking patient. Frequently in our experience, their desire to seek rhinoplasty is influenced by a deterioration in function, rather than solely dictated by improvement in aesthetics. According to Janeke and Wright, there are four areas involved in normal tip support: the fibrous connection between the upper and lower lateral cartilages (scroll area); the sesamoid complex that attached the lateral crura to the pyriform aperture; the fibrous connections between the domes of published online

February 25, 2021
Issue Theme Facial Rejuvenation: A Multidimensional and Multimodal Perspective; Guest Editor: Fred G. Fedok, MD, FACS (c) 2021. Thieme. All rights reserved. Thieme Medical Publishers, Inc., 333 Seventh Avenue, 18th Floor, New York, NY 10001, USA
DOI https://doi.org/ 10.1055/s-0041-1725106. ISSN 0736-6825. 


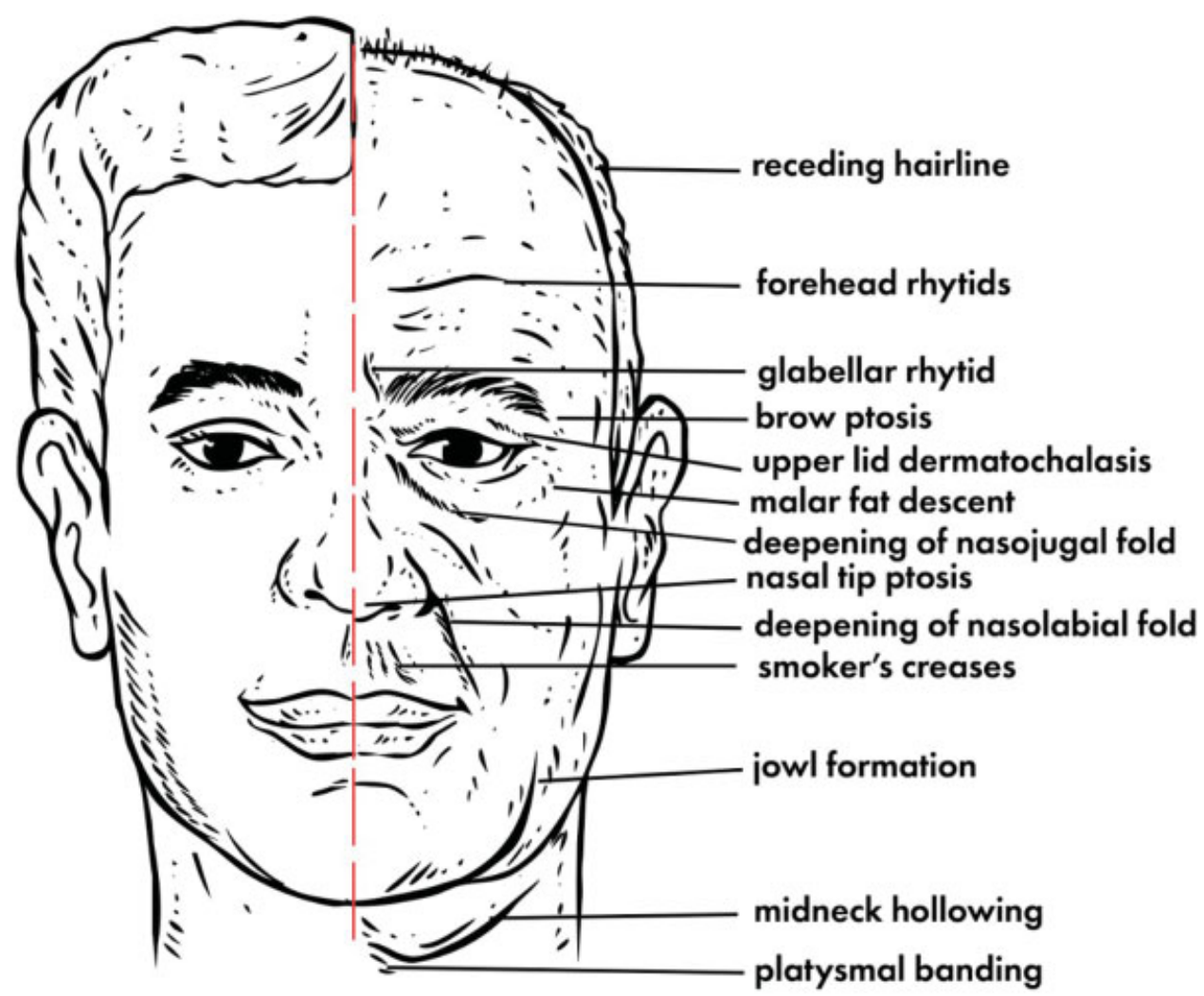

Fig. 1 Characteristics of the aging nose.

the lower lateral cartilages; and the attachments of the medial crural footplates to the caudal septum. ${ }^{7}$ With aging, these support mechanisms all weaken giving rise to distinctive changes to the aging nose. ${ }^{8}$ Numerous studies have identified key hallmarks in the aging nose. ${ }^{8-11}$ For example, the effect of aging on the scroll area, leads to a separation between the upper and lower lateral cartilages which in turn leads to marked tip ptosis and a hanging columella (-Fig. 1). Whilst the nasal bony pyramid itself does not necessarily shift in position, the bone quality becomes more brittle and so care must be taken when performing osteotomies. Skin over this area tends to become thinner with age, highlighting bony irregularities; more caudally it tends to thicken and in turn further down-rotates the tip. Functionally too, as the lower lateral cartilages and dilator naris weaken with age, external nasal valve collapse becomes more apparent. ${ }^{8}$

As a result, the correction of apparent facial asymmetries, especially in the aging face, poses a test for even the most seasoned of rhinoplasty surgeons. The present article illustrates the authors' experience in the management of restoring balance and symmetry to the aging nose.

\section{Assessment of the Nose and Its Relation to the Face}

Prior to commencing any examination, accurate assessment of the nose in relation to the face begins with accurate history taking. To begin with, it is important to gauge whether there are any functional nasal disorders such as nasal obstruction, altered sense of smell, or other symptoms of rhinological disease. History of previous trauma or nasal or facial surgery is helpful to manage expectations. For some patients they may have a history of congenital facial abnormalities such as a cleft lip and/or palate and may have undergone multiple previous surgical procedures. In such patients in particular, the face itself is frequently asymmetric.

In the aging patient cohort, these apparent facial asymmetries may develop with time. Whilst traditional focus has always been on soft tissue remodeling of the face, it has been shown that bony resorption of the facial skeleton does also take place, most notably in the maxilla. ${ }^{12}$ This resorption can take place at differing rates, thus exaggerating facial asymmetry. For example, around the periorbita, the orbital aperture increases in size with age due to bony resorption, but this may take place at differing rates between both sides of the face. ${ }^{13}$ Within the midface, the maxilla tends to resorb more quickly than the zygoma. $^{14}$

During the consultation, patient expectations must be explored in depth, and may necessitate several meetings. We recommend asking the patient what they feel requires improving. It is not uncommon for patients seeking rhinoplasty surgery to be unaware of facial asymmetry and without accurate assessment, any post-surgical structural improvement to the nose may be less apparent to the patient. ${ }^{15}$

In patients with an aging nose, further considerations must be undertaken as to what is realistically attainable. It is essential to communicate these with the patient and we recommend asking the patient the single biggest improvement that they wish to make about their nose. In the authors' experience, aging rhinoplasty surgery necessitates a greater 


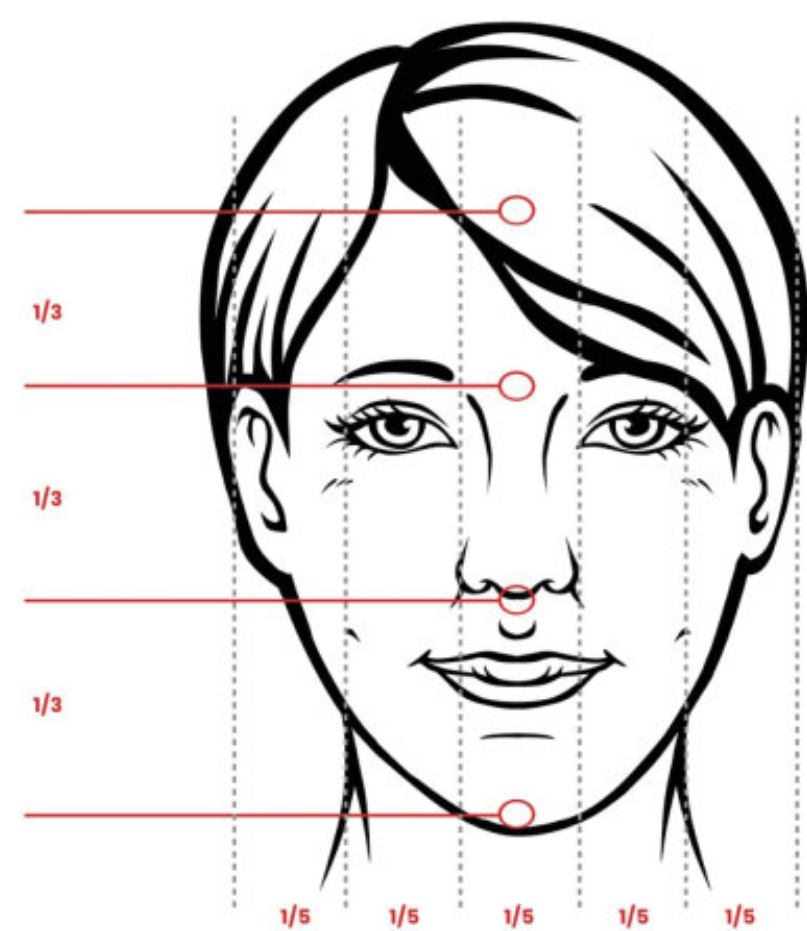

Fig. 2 Ideal facial proportions. reliance on structural grafting rather than suture techniques for the reasons outlined. The techniques utilized for younger patients may not be applicable and thus expectations must be realistic with complete restoration of facial symmetry almost never possible.

\section{Clinical Examination}

Following a thorough history, it is at this stage we recommend performing a detailed nasofacial analysis. This assessment should be performed systematically in order to identify deformities, evaluate anatomical relationships, and establish goals for surgery. ${ }^{16}$ Facial asymmetries can be easily picked up at this stage by the experienced rhinoplasty surgeon. At all times, the surgeon will also be taking in to account the patient's expectations and perceived aesthetic irregularities. Standard rhinoplasty views as per the Institute of Medical Illustrators (IMI) should be captured. ${ }^{17}$ These photographs can prove to be an excellent adjunct to the consultation with the patient.

First, it is important to assess the nose endonasally with both anterior rhinoscopy and endoscopically. This will highlight any gross septal deviations which in turn frequently give rise to an externally deviated appearance. Next, the nose is systematically assessed from a frontal, lateral, basal, and

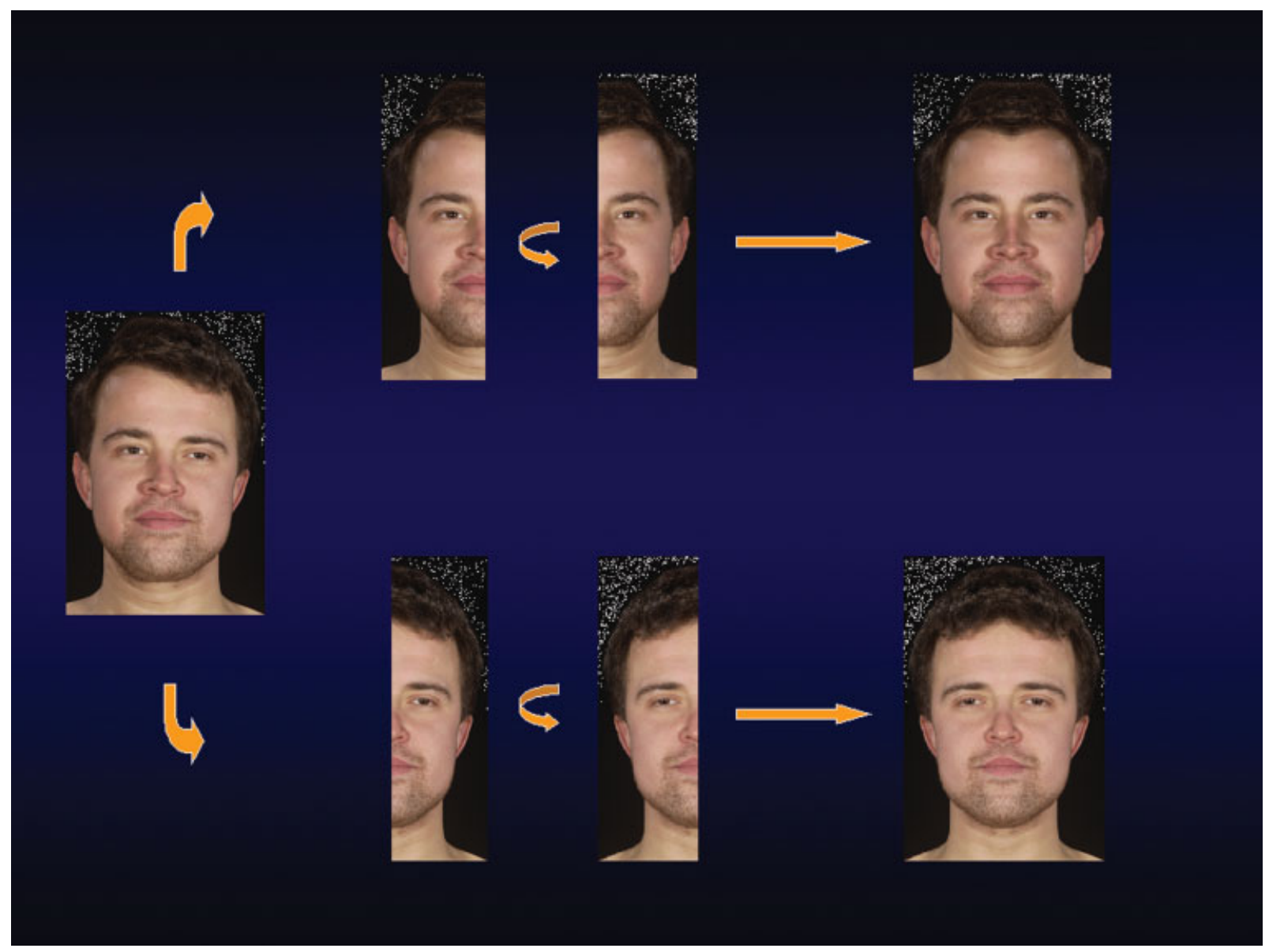

Fig. 3 An illustration of significant facial and nasal asymmetry. Note the difference in the width and height of the alar margins, orbits, and mandibles (left). When the face is divided in halves and both sides are mirror imaged (middle) then put together, two different facial appearances are obtained (right). 

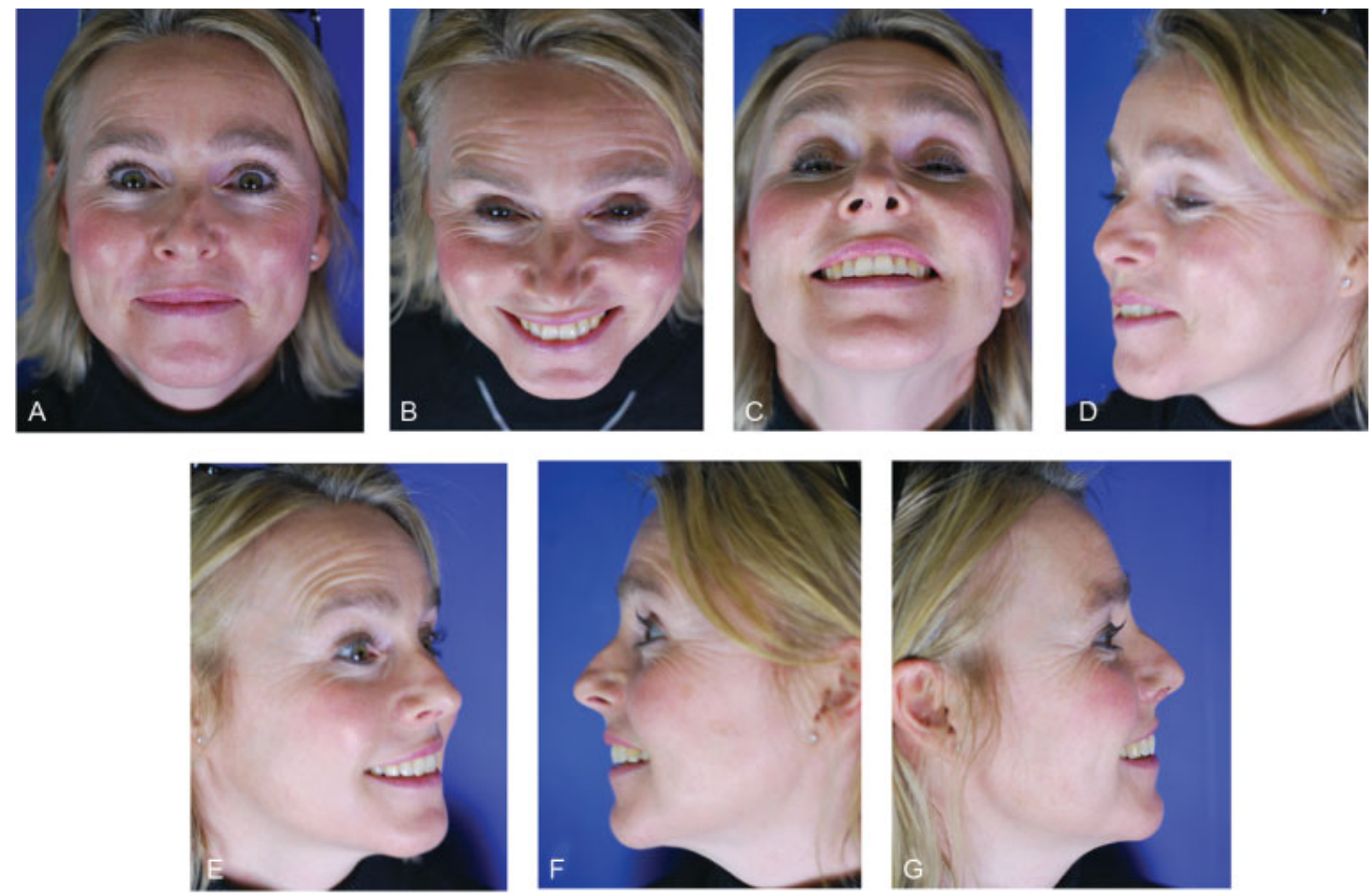

Fig. 4 A 51-year-old patient with orbital, alar base, mandibular asymmetry, and crooked nose.
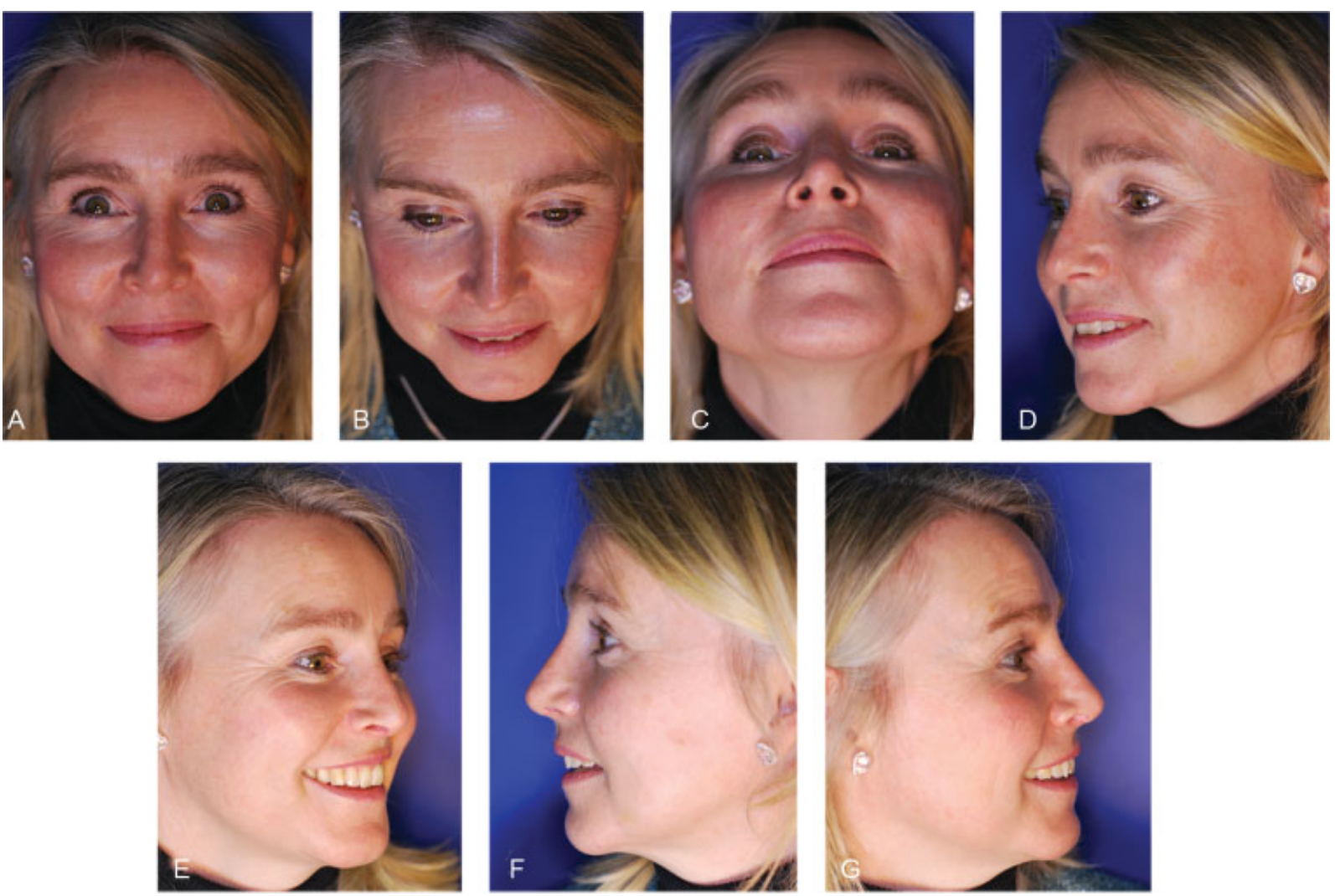

Fig. 5 Patient in -Fig. 4 following rhinoplasty with improved nasal appearance and perception of facial asymmetry. 
skyline view, paying particular attention to its position with the rest of the face.

The frontal view in particular will give details of any facial asymmetries. The ideal facial proportions consist of dividing the face into equal vertical fifths and horizontal thirds; vertical landmarks being the outer edge of the pinna, the lateral and medial canthus of the eyes; horizontal landmarks being the trichion, subnasale, and mentum (-Fig. 2). The facial midline is constructed using two main landmarks. In a symmetrical face, a vertical line dropping from the glabella to midpoint of the chin should pass through the mid-philtrum of the upper lip (Cupid's bow). Mandibular asymmetry too, such as through overeruption of unilateral maxillary dentition, can be picked up here giving rise to an asymmetric face. ${ }^{18}$ Focusing then on the nose, any deviation of the upper, middle, and lower third of the nose is assessed. Care should be taken to appreciate the dorsal aesthetic lines, nasal tip, alar rims, and alar base. Indeed, Chatrath et al found that the most significant marker of facial asymmetry in rhinoplasty patients is the distance between the midline and lateral alar margin as well as the height of the alar base (-Fig. 3). ${ }^{2}$

Lateral views of the face help tremendously with assessment of nasal projection. Photos ought to be taken in the Frankfort plane-an imaginary line from the external auditory canal to infraorbital rim. In the authors' opinion, all too frequently, patients mistakenly identify overprojected noses when in fact, there is either maxillary hypoplasia or mandibular retrognathia. Indeed, midface hypoplasia is characterized on lateral view with a flattened upper lip and an obtuse nasolabial angle. In these patients, it may be helpful to consult with an oral and maxillofacial surgeon too. Standard rhinoplasty assessments can then be carried out to assess the nasal dorsum, supratip, tip rotation, nasofrontal, and nasolabial angles. When assessing true nasal projection, Goode's ratio should be used. It is essential to compare both lateral views as the alar-columellar relationship may be different from each side.

Congenitally twisted medial crura are best assessed via the basal view which can in turn give rise to nostril asymmetry. Caudal septal dislocations, previous scars, and columella-tolobule ratio are all best picked up on basal view.

\section{Corrective Surgery}

Following detailed history, examination, and photo assessment, it is recommended that any identified facial asymmetries are clearly explained to patients. Prior to embarking on surgery, there has to be a degree of concordance between patient perception and surgeon assessment. Expectations must be realistic, and an understanding should prevail that despite successful correction of any nasal abnormality, perceived facial asymmetry may persist. This may be in part due to a newly detected disharmony between the straightened nose and the pre-existing asymmetric face. Indeed, Hafezi et al found that $74 \%$ of patients with a deviated nose had significant facial asymmetry and a severely crooked nose was associated with a major disharmony of the face. ${ }^{19}$

Conversely, several studies have shown that even in the context of asymmetric faces, successful improvement of nasal symmetry can give rise to a perception of better facial symmetry. ${ }^{2,6}$ Moreover, Yi and Jang showed that regardless of the type of nasal deviation, facial asymmetry was more commonly found in patients with deviated noses compared to a control group. ${ }^{1}$ It is the authors' opinion that rhinoplasty for facial asymmetry is by no means a contraindication to surgery but clear communication must take place between surgeon and patient in order to manage expectations.

\section{Special Considerations for the Ageing Nose}

In the aging nose, it is worth bearing in mind that more extensive soft tissue dissection may be required when performing rhinoplasty. The skin and soft tissue envelope is more inelastic and in addition, any contour irregularities become more visible when the skin is redraped. Although cartilage quality tends to deteriorate with age, it is the authors' opinion that structural grafting techniques are still preferable to suture techniques alone. Where there is insufficient strong septal cartilage, irradiated cadaveric costal cartilage is our preference rather than autologous costal cartilage. With advancing age, costal cartilage tends to ossify rendering it suboptimal for use in rhinoplasty. Auricular cartilage too, does not provide enough structural support. - Figs. 4 and $\mathbf{5}$ show a 51-year-old patient with moderate facial asymmetry before and after rhinoplasty, respectively. Following surgery, there is a perceived improvement in facial asymmetry.

\section{Conclusion}

The restoration of symmetry in the aging nose requires a thorough understanding of the changes that take place in the facial skeleton and soft tissues. Techniques that may be suitable for younger patients may not be applicable for the aging nose. Grafting with strong autologous septal cartilage or irradiated cadaveric costal cartilage is preferred rather than the use of ossified autologous costal and/or auricular cartilage. Above all, rhinoplasty in the asymmetric aging face can improve perceived facial symmetry but patient expectations must be realistic and surgeon assessment precise.

\section{Funding \\ None.}

\section{Conflict of Interest}

None declared.

\section{References}

1 Yi JS, Jang YJ. Frequency and characteristics of facial asymmetry in patients with deviated noses. JAMA Facial Plast Surg 2015;17(04): 265-269

2 Chatrath P, De Cordova J, Nouraei SA, Ahmed J, Saleh HA. Objective assessment of facial asymmetry in rhinoplasty patients. Arch Facial Plast Surg 2007;9(03):184-187

3 Carvalho B, Ballin AC, Becker RV, Berger CA, Hurtado JG, Mocellin M. Rhinoplasty and facial asymmetry: analysis of subjective and anthropometric factors in the Caucasian nose. Int Arch Otorhinolaryngol 2012;16(04):445-451 
4 Zaidel DW, Cohen JA. The face, beauty, and symmetry: perceiving asymmetry in beautiful faces. Int J Neurosci 2005;115(08): $1165-1173$

5 International Society of Aesthetic Plastic Surgery (ISAPS) international survey on aesthetic/cosmetic procedures performed in 2018. Accessed November 27, 2020 at: https://www.isaps.org/wp-content/uploads/2020/10/ISAPS-Global-Survey-Results-2018-1.pdf

6 Nouraei SA, Pulido MA, Saleh HA. Impact of rhinoplasty on objective measurement and psychophysical appreciation of facial symmetry. Arch Facial Plast Surg 2009;11(03):198-202

7 Janeke JB, Wright WK. Studies on the support of the nasal tip. Arch Otolaryngol 1971;93(05):458-464

8 Romo T III, Soliemanzadeh P, Litner JA, Sclafani AP. Rhinoplasty in the aging nose. Facial Plast Surg 2003;19(04):309-315

9 Krmpotić-Nemanić J, Kostović I, Rudan P, Nemanić G Morphological and histological changes responsible for the droop of the nasal tip in advanced age. Acta Otolaryngol 1971;71(02):278-281

10 Ries WR, Rathfoot CJ. The aging nose in rhinoplasty for facial rejuvenation. Facial Plast Surg 1996;12(02):197-203

11 Toriumi DM. Surgical correction of the aging nose. Facial Plast Surg 1996;12(02):205-214
12 Mendelson B, Wong $\mathrm{CH}$. Changes in the facial skeleton with aging: implications and clinical applications in facial rejuvenation. Aesthetic Plast Surg 2012;36(04):753-760

13 Kahn DM, Shaw RB Jr. Aging of the bony orbit: a three-dimensional computed tomographic study. Aesthet Surg J 2008;28(03):258-264

14 Flowers RS. Periorbital aesthetic surgery for men. Eyelids and related structures. Clin Plast Surg 1991;18(04):689-729

15 Gunter JP, Rohrich RJ. Management of the deviated nose. The importance of septal reconstruction. Clin Plast Surg 1988;15(01):43-55

16 Rohrich RJ, Villanueva NL, Small KH, Pezeshk RA. Implications of facial asymmetry in rhinoplasty. Plast Reconstr Surg 2017;140 (03):510-516

17 IMI National Guidelines. A guide to good practice: rhinoplasty and septorhinoplasty. 2019. Accessed November 27, 2020 at: https:// www.imi.org.uk/wp-content/uploads/2019/03/2019_Mar_IMINatGuidelines_Rhinoplasty_V2x-1.pdf

18 Naini FB, Gill DS. Facial aesthetics: 2. Clinical assessment. Dent Update 2008;35(03):159-162, 164-166, 169-170

19 Hafezi F, Naghibzadeh B, Nouhi A, Yavari P. Asymmetric facial growth and deviated nose: a new concept. Ann Plast Surg 2010;64 (01):47-51 